\title{
COINCIDENCE OF NODES FOR GENERALIZED CONVEX FUNCTIONS
}

\author{
BY \\ R. M. MATHSEN
}

In a recent paper [1] I. B. Lazarevic announced an extension of results of L. Tornheim [2; Theorems $2 \& 3$ ] concerning points of contact between two distinct members of an $n$-parameter family and between a member of an $n$-parameter family and a corresponding convex function. In the proofs of these extensions [1; Theorems $3.1 \&$ 3.2] use is made of Tornheim's Convergence Theorem [2; Theorem 5]; however this theorem is not correctly applied in [1] since it requires distinct limiting nodes, and that hypothesis necessarily fails in the approach used in [1]. In this note proofs of results more general than those in [1] are given independent of convergence theorems. Throughout this note $F \subset C^{r}(I)$ for $r \geq 0$ and $I$ is an interval of the reals. Let $\lambda(n)=\left(\lambda_{1}, \lambda_{2}, \ldots, \lambda_{k}\right)$ where $\lambda_{1}, \ldots, \lambda_{k}$ and $k$ are positive integers satisfying $\lambda_{1}+\lambda_{2}+\cdots+\lambda_{k}=n$. $F$ is said to be a $\lambda(n)$-parameter family on $I$ in case for every choice of $k$ points $x_{1}<x_{2}<\cdots<x_{k}$ in $I$ and every set $\left\{y_{i}^{j}\right\}$ of $n$ real numbers there is a unique $f \in F$ satisfying $f^{(j)}\left(x_{i}\right)=y_{i}^{j}, j=0,1, \ldots, \lambda_{i}-1, i=$ $1,2, \ldots, k$. A function $g$ is said to be $\lambda(n)$-convex with respect to $F$ on $I$ in case for every choice of $k$ points $x_{1}<x_{2}<\cdots<x_{k}$ from $I$ and every $f$ in $F$ satisfying $f^{(j)}\left(x_{i}\right)=g^{(j)}\left(x_{i}\right)$ for $j=0,1, \ldots, \lambda_{i}-1, i=1,2, \ldots, k$, we have

$$
(-1)^{M(i)}(g(x)-f(x)) \geq 0 \text { when } \quad x_{i-1}<x<x_{i}
$$

for $i=2,3, \ldots, k$ where $M(i)=n+\lambda_{1}+\cdots+\lambda_{i-1}$.

In the case that $\lambda_{1}=\lambda_{2}=\cdots=\lambda_{k}=1$ we call a $\lambda(n)$-parameter family an $n$-parameter family and a $\lambda(n)$-convex function is called an $n$-convex function. Replacing $\geq$ by $\leq$ in (1) replaces convex by concave in the definition.

Definition. Functions $f$ and $g$ defined on an interval $I$ are said to graze (or have a point of contact) at an interior point $z$ of $I$ if $f(z)=g(z)$ and there is a $d>0$ so that $f(x)-g(x)$ is of constant sign for $0<|x-z|<d$.

THEOREM 1. Let $g$ be convex and $h$ be concave with respect to the $n$-parameter family $F$ on an interval $I$ of the real numbers. If $g$ and $h$ graze at $k$ points and $g-h$ changes sign at $m$ points in $I$, then $2 k+m \leq n$ unless $g$ and $h$ are identical on a subinterval of $I$. Moreover, if $h, g \in F$, then $2 k+m<n$.

Received by the editors July 6, 1978 and, in revised form, Febuary 20, 1979. 
The proof of this theorem consists of showing it first for $g$ and $h$ both in $F$, then for only one in $F$, and finally for neither in $F$. Because of the similarity of these arguments, only the first case will be considered here.

We remark that in the case where $2 k+m=n$ we may conclude that $g-h>0$ to the right of the last zero of $g-h$ in $I$ and $(-1)^{n}(g-h)>0$ to the left of the first zero of $g-h$ in $I$. Hence the sign of $g-h$ between its zeros is dependent only on the relative positions of these zeros and whether or not they are points of contact for $g$ and $h$.

Proof. The case $k=1$ is Theorem 3 in [2], and this same theorem resolves the cases $n=2$ and $n=3$. We assume the theorem is true for $n-1$ in place of $n$, and show that it is then true for $n$. Let $f_{1}$ and $f_{2}$ graze at points $z_{1}<z_{2}<$ $\cdots<z_{k}$ in $I$, and let $f_{1}-f_{2}$ change sign at $x_{1}<x_{2}<\cdots<x_{m}$ in $I$. Suppose that $m+2 k>n$. First observe that $z_{1}<x_{1}$. For if not, we could consider the $n$-1-parameter family $G$ consisting of all $f \in F$ with $f\left(x_{1}\right)=f_{1}\left(x_{1}\right)$ restricted to the interval $I \cap\left(x_{1}, \infty\right)$. Then $f_{1}$ and $f_{2}$ are in $G$ and graze at $k$ points, while $f_{1}-f_{2}$ changes sign at $m-1$ points. Hence $2 k+m-1 \geq n-1$ contracting our induction assumption. Similarly for $z_{k}>x_{m}$ and for $m+2 k>n$. Thus we can and do assume that $m+2 k=n$.

Let $f_{1}-f_{2}$ have zeros $z_{1}=y_{1}<y_{2}<\cdots<y_{j}=z_{k}$ where $j=k+m$. Pick $u<y_{1}$ and $v>y_{j}$. Pick $f \in F$ so that $f\left(y_{i}\right)=f_{2}\left(y_{i}\right)$ for $i=1,2, \ldots, j, f(u)=f_{1}(u)$, and $f(v)=f_{2}(v)$. In addition if $f_{1}$ and $f_{2}$ graze at $y_{i}$ for $1<i<j$, pick $u_{i}$ between $y_{i}$ and $y_{i+1}$ and let $f\left(u_{i}\right)=f_{2}\left(u_{i}\right)$. Then $f$ is specified at $j+2+k-2=m+2 k=n$ points and so is uniquely determined. Also $f-f_{2}$ has $n-1$ zeros, and so it changes sign at each of these zeros. We shall without loss of generality assume that $f_{2}(v)<f_{1}(v)$. There are two cases to consider:

CAse 1. $f(x)<f_{2}(x)$ for $z_{k}<x<v$.

CASE 2. $f(x)>f_{2}(x)$ for $z_{k}<x<v$. We consider Case 1 first. $f(x)-f_{2}(x)$ and $f_{1}(x)-f_{2}(x)$ have opposite signs for $z_{k}<x<v . f_{1}(x)-f_{2}(x)$ changes sign $m$ times for $u<x<v$, and $f(x)-f_{2}(x)$ changes signs $m+2(k-2)$ times for $u<x<v$. So $f_{1}(x)-f_{2}(x)$ and $f(x)-f_{2}(x)$ have opposite signs for $u<x<z_{1}$. This contradicts $f_{1}(u)=f(u)$. Next for Case $2 f(x)-f_{2}(x)$ and $f_{1}(x)-f_{2}(x)$ have the same sign for $z_{k}<x<v$. Either $f(x)-f_{1}(x)$ has a zero for $z_{k}<x<v$ or else $f$ and $f_{1}$ graze at $z_{k} . f-f_{2}$ changes sign at $z_{k}$ and $f_{1}-f_{2}$ does not. Thus $f-f_{2}$ and $f_{1}-f_{2}$ have opposite signs in a small interval with right endpoint $z_{k}$. Next pick the largest $i<j$ for which $f_{1}$ and $f_{2}$ graze at $y_{i}$. If $i=j-1, f-f_{2}$ changes sign at $u_{i}$ implies that $f(x)-f_{1}(x)$ has a zero for $y_{i}<x<u_{i}$ or else $f$ and $f_{1}$ graze at $y_{i}$. Also $y_{i}$ is the right endpoint of an interval on which $f-f_{2}$ and $f_{1}-f_{2}$ have the same sign. If $i<j-1, f-f_{2}$ and $f_{1}-f_{2}$ both change sign at $y_{q}$ for $i<q<j$, and hence $f-f_{2}$ and $f_{1}-f_{2}$ have the same sign in an interval with right endpoint $y_{q}$. The above argument can be applied to show that for each $y_{i}$ for $i>1$ at which $f_{1}$ and $f_{2}$ 
graze either $f$ and $f_{1}$ graze at $y_{i}$ or else $f(x)-f_{1}(x)$ has a zero for $y_{i}<x<u_{i}$. Thus twice the number of points of contact of $f$ and $f_{1}$ plus the number of zeros of $f-f_{1}$ (not counting $u$ ) which are not points of contact is at least as large as $m+2 k-1=n-1 . f-f_{1}$ has a zero at $u$, and this is impossible as pointed out previously when we observed that $z_{1}<x_{1}$. Hence the theorem is established.

In the case of $n$-convex functions, if (1) holds for some fixed $i, 1 \leq i \leq k+1$, then it holds for every $i$ in that range. In the case of coincidence of nodes, i.e., $\lambda_{i}>1$ for at least one $i$, a similar result holds if

$$
\lambda_{i} \leq 2 \text { for all } i=1,2, \ldots, k .
$$

By $[\lambda(n)]$ we shall mean $\lambda(n)$ together with the set of all ordered partitions $\mu(n)$ obtained from $\lambda(n)$ by replacing a 2 in $\lambda(n)$ by two 1's. See [3, page 39].

THEOREM 2. Let $F$ be a $\mu(n)$-parameter family for all $\mu(n)$ in $[\lambda(n)]$. Assume (6). If (1) holds for a fixed $i$ between 1 and $k+1$ inclusive, then it holds for every $i$ in this range. Moreover there is no distinction between $\lambda(n)$ - and $\lambda(n)^{*}$ convexity in this case. See [3, page 37].

This theorem is an immediate consequence of the lemma that follows.

LEMMA. Let $F$ be a $(1,1)$-parameter and a (2)-parameter family on the open interval $I$. Let $g$ be a differentiable real valued function defined on $I$ having the property that if $g\left(x_{0}\right)=f\left(x_{0}\right)$ and $g^{\prime}\left(x_{0}\right)=f^{\prime}\left(x_{0}\right)$ for some $f$ in $F$ and some $x_{0}$ in $I$, then

$$
g(x) \geq f(x)
$$

whenever $x$ is in $I$ and

$$
x \geq x_{0} .
$$

Then $g$ is convex with respect to $F$ on I and, for $f$ as above, (1) holds for all $x$ in $I$.

Proof. Suppose $g$ is not convex. Then there are points $x_{1}<x_{2}$ in $I$ and a function $f$ in $F$ so that $f\left(x_{1}\right)=g\left(x_{1}\right), f\left(x_{2}\right)=g\left(x_{2}\right)$, and $f(x)<g(x)$ for $x_{1}<x<$ $x_{2}$. Consider the cases (i) $f^{\prime}\left(x_{1}\right)<g\left(x_{1}\right)$, and (ii) $f^{\prime}\left(x_{1}\right)=g^{\prime}\left(x_{1}\right)$. In case (i) pick $h \in F$ so that $h\left(x_{1}\right)=g\left(x_{1}\right)$ and $h^{\prime}\left(x_{1}\right)=g^{\prime}\left(x_{1}\right)$. Then $h(x) \leq g(x)$ for $x \geq x_{1}$, and since $h(x)>f(x)$ for $x$ near and $>x_{1}, f$ and $h$ must intersect in $\left(x_{1}, x_{2}\right)$. This contradiction shows that the case (i) is impossible. In case (ii) pick a point $u$ between $x_{1}$ and $x_{2}$. We get an immediate contradiction by considering $h \in F$ satisfying $h\left(x_{1}\right)=g\left(x_{1}\right), h(u)=g(u)>f(u)$. This shows that $g$ is convex. Suppose that $f(u)>g(u)$ for some point $u$ of $I$ with $u<x_{0}$. Then $f(x)>g(x)$ for all $x<x_{0}$. The function $h$ in $F$ satisfying $h\left(x_{0}\right)=g\left(x_{0}\right)$ and $h(u)=g(u)$ must satisfy $h^{\prime}\left(x_{0}\right)=f^{\prime}\left(x_{0}\right)$ which is not possible.

Clearly if (2) is replaced by

$$
x \leq x_{0}
$$


then the same result follows. Also if (1) is replaced by $g(x) \leq f(x)$, then $g$ is concave with repsect to $F$ whether or not (2) is replaced by (2)'.

\section{REFERENCES}

1. I. B. Lazarevic, Some Properties of $n$-parameter Families of Functions, Univ. Beograd. Publ. Elektrotehn. Fak. Ser. Mat. Fiz. No. 357-380 (1971), 101-106 MR47\#8792.

2. L. Tornheim, On n-parameter families of functions and associated convex functions, Trans. Amer. Math. Soc. 69 (1950), 457-467. MR12\#395.

3. R. M. Mathsen, $\lambda(n)$-convex functions, Rocky Mountain Journal of Math. 2(1) (1972), 31-43. MR45\#3651.

Department of Mathematics

NORTH Dakota STATE UNIVERSTTY

FARGO, NORTH DAKOTA 58102

and

UNIVERSTTY OF AlBERTA

Edmonton, Alberta

T6G 2G1 